BULL. AUSTRAL. MATH. SOC.

\title{
CENTRAL COMMUTATORS
}

\section{A. CARANTI AND C.M. SCOPPOLA}

\begin{abstract}
We give examples of finite groups of odd prime power order in which the commutators lying in the centre do not generate the intersection of the centre and the commutator subgroup.
\end{abstract}

The problem of finding a finite $p$-group $G$ with commutator subgroup $G_{2}$ and centre $Z(G)$ so that $G_{2} \cap Z(G)$ is not generated by commutators has been considered by $R$. Oliver, and communicated to the authors by J. Brandt.

To our knowledge, no group having this property has been explicitly recorded in the literature. The example we propose hereafter is meant to display the ideas involved in the construction of it; examples of smaller order, however, can be found, as remarked at the end of this paper.

Notation follows [1]. We use freely elementary commutator calculus as in III.1 of [1]. Matrices act on vectors from the right; that is, vectors are regarded as row vectors.

Let $p$ be an odd prime number. Let $E$ be an elementary abelian group of order $p^{3}$, viewed as a vector space over $G F(p)$, and let

$$
e_{1}, e_{2}, e_{3}
$$

be a base of $E$. Let $y$ be the automorphism of $E$ given, with respect to

Received 6 February 1984.

Copyright Clearance Centre, Inc. Serial-fee code: 0004-9727/84 $\$ A 2.00+0.00$ 
the given base, by the matrix

$$
\left[\begin{array}{lll}
1 & 1 & \\
& 1 & 1 \\
& & 1
\end{array}\right]
$$

since $p>2, y$ has order $p$. Then the semidirect product $H=E\langle y\rangle$ is a group of order $p^{4}$, and of nilpotency class 3 . Indeed, if we set $x=e_{1}$, we get

(1)

$$
\left\{\begin{array}{l}
{[x, y]=e_{2},[x, y, y]=e_{3},} \\
H_{2}=[H, H]=\left\langle e_{2}, e_{3}\right\rangle, H_{3}=\left[H_{2}, H\right]=\left\langle e_{3}\right\rangle=Z(H) .
\end{array}\right.
$$

$H$ is group 12 of Satz III.12.6 of [1] (note that $H$ has exponent $p$ for $p>3$, while $H$ has exponent 9 for $p=3$, check [1, Aufgabe 29, p. 349].

Let now $A$ be an elementary abelian $p$-group of order $p^{10}$, viewed as a vector space over $G F(p)$, and let

$$
a_{1}, a_{2}, a_{3}, c_{2}, c_{3}, b_{1}, b_{2}, b_{3}, d_{2}, d_{3}
$$

be a base of $A$. Consider the elements $\xi, \eta \in A u t(A)$ given, with respect to the given base, in matrix form by

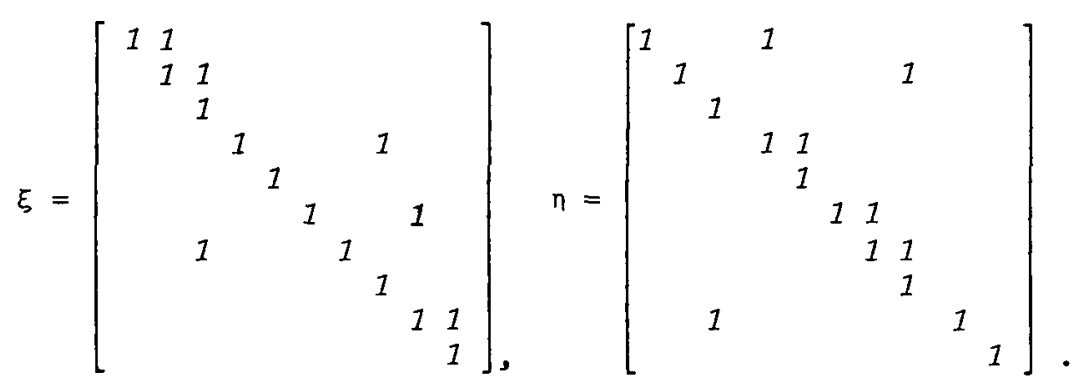

A straightforward computation gives

$$
\xi n=n \xi ;
$$

since $\xi$ and $\eta$ are unipotent, their Jordan forms have blocks of size at 
most 3 , and $p>2$, we have

$$
\xi^{p}=n^{p}=1
$$

since $\left\langle a_{1}, a_{2}, a_{3}\right\rangle$ is invariant under $\xi$ but not invariant under $n$, $\eta$ is not a power of $\xi$. Therefore $\langle\xi, n\rangle$ is an elementary abelian subgroup of $A u t(A)$ of order $p^{2}$. Then setting $p(x)=\xi, p(y)=n$, a homomorphism $\rho: H \longrightarrow \operatorname{Aut}(A)$ is defined, with

$$
\operatorname{ker}(\rho)=H_{2}
$$

Let $G$ be the semidirect product of $A$ and $H$ relative to $\rho$, so that $|G|=p^{14}$. Set $a_{1}=a, b_{1}=b$; then in $G$

(4)

$$
\begin{aligned}
& \left\{\begin{array}{l}
a_{2}=[a, x] \\
a_{3}=[a, x, x]=[b, y, x]=[b, x, y] \\
c_{2}=[a, y] \\
c_{3}=[a, y, y]
\end{array}\right. \\
& \left\{\begin{array}{l}
b_{2}=[b, y] \\
b_{3}=[b, y, y]=[a, x, y]=[a, y, x] \\
d_{2}=[b, x] \\
d_{3}=[b, x, x] .
\end{array}\right.
\end{aligned}
$$

Furthermore, it is easy to see that $a_{3}, b_{3}, c_{3}, d_{3}$ are left fixed by $\xi$ and $n$, and then

$$
a_{3}, b_{3}, c_{3}, d_{3} \in Z(G)
$$

Since $A \triangleleft G,[A, H] \leqq A ;$ by $(2)$,

$$
\left[A, H_{2}\right]=1 \text {, }
$$

and then $\mathrm{AH}_{2}=\mathrm{A} \times \mathrm{H}_{2}$; therefore, by easy commutator identities

$$
G_{2}=[G, G]=[A H, A H]=[A, H] H_{2}=[A, H] \times H_{2}
$$

and then, by (1), $G_{2}$ is the 10 -dimensional direct sum of 


$$
[A, H]=\left\langle a_{2}, a_{3}, c_{2}, c_{3}, b_{2}, b_{3}, d_{2}, d_{3}\right\rangle
$$

and

$$
H_{2}=\langle[x, y],[x, y, y]\rangle
$$

By (6) and the above $\left[G_{2}, A\right]=1$; then

$$
\begin{aligned}
G_{3} & =\left[G_{2}, G\right]=\left[G_{2}, A H\right]=\left[G_{2}, H\right]= \\
& =\left[[A, H] H_{2}, H\right]=[A, H, H] H_{3}=[A, H, H] \times H_{3},
\end{aligned}
$$

and thus, by (1),

$$
G_{3} \text { is the } 5 \text {-dimensional direct sum of }[A, H, H]
$$

$$
=\left\langle a_{3}, b_{3}, c_{3}, d_{3}\right\rangle \text { and } H_{3}=\langle[x, y, y]\rangle \text {. }
$$

By (5), (1) and (6) $G_{3} \leq Z(G)$ and $G$ has class 3 . Consider now the element $g=[a, x][b, y]^{-1} \in G_{2} ; \quad$ (3) and (4) yield

$$
[g, x]=[a, x, x,][b, y, x]^{-1}=1,[g, y]=[a, x, y][b, y, y]^{-1}=1 \text {. }
$$

Since $A$ is abelian, and $G=\langle A, x, y\rangle$, we obtain $g \in Z(G) \cap G_{2}$; by (7), however, $g \notin G_{3}$. We now show that all central commutators of $G$, i.e. the commutators of $G$ lying in $Z(G)$, are in fact in $G_{3}$. This will imply that $g$ is not a product of central commutators. Suppose

$$
w=\left[g_{1}, g_{2}\right] \in Z(G), g_{1}, g_{2} \in G
$$

It is enough to show $w \equiv 1\left(\bmod G_{3}\right)$. For a suitable $2 \times 4$ matrix $t=\left[t_{i j}\right]$, with entries in $\mathbb{Z}$ we have, $\bmod G_{3}$ '

$$
\begin{aligned}
w & \equiv\left[a^{t_{11}} b^{t_{12}} x^{t_{13}} y^{t_{14}}, a^{\left.t_{21} b^{t_{22}} t^{t_{23}} y t_{24}\right]}\right. \\
& \equiv[a, x]^{m_{13}}[a, y]^{m_{14}}[b, x]^{m_{23}}[b, y]^{m_{24}}[x, y]^{m_{34}},
\end{aligned}
$$

where $m_{i j}=t_{1 i} t_{2 j}-t_{1 j} t_{2 i}$. Since congruences are $\bmod G_{3}$, and $G_{3} \leqq Z(G)$ we get, by (8), (3) and (4),

$$
1=[w, y]=b_{3}^{m_{13+m_{24}}} c_{3}^{m_{14}} a_{3}^{m_{23}}[x, y, y]^{m_{34}} \text {. }
$$


Therefore by (7) we have, $\bmod p, m_{13}+m_{24} \equiv 0, m_{14} \equiv m_{23} \equiv m_{34} \equiv 0$. Now the well known relation

$$
m_{12} m_{34}-m_{13} m_{24}+m_{14} m_{23}=0
$$

holds among the $2 \times 2$ minors of $t$ (see for instance [1], Beispiel III.1.12); we obtain $m_{i j} \equiv 0$ for $\{i, j\} \neq\{1,2\}$, and thus, by (9), $w \equiv 1\left(\bmod G_{3}\right)$, as required.

Note that an example of smaller order can be obtained by adding to a presentation of $G$ as above the relation $[b, x, x]=1$. It is conceivable that examples of even smaller order exist.

\section{Reference}

[1] B. Huppert, Endliche Gmuppen I (Springer, Berlin, 1967).

Department of Mathematics,

University of Trento,

I-38050 Povo (Trento),

ITALY. 